Original Article

Joumal of Epilepsy Research pISSN 2233-6249 / eISSN 2233-6257

\title{
Relative Safety of Different Antidepressants for Treatment of Depression in Chronic Epileptic Animals Associated with Depression
}

\author{
Rakesh Kumar Sharma, Tanveer Singh, Awanish Mishra, Rajesh Kumar Goel \\ Department of Pharmaceutical Sciences and Drug Research, Punjabi University, Patiala, Punjab, India
}

Background and Purpose: Depression is one of the major psychiatric comorbidities associated with epilepsy. The inconclusive results of antidepressants (ADs) regarding their safety in regard to convulsions have strongly contributed towards under treatment of depression in people with epilepsy (PWE). Thus, the present study was envisaged to assess the relative safety of four different classes of ADs regarding their convulsive potential in kindled/epileptic animals.

Methods: Kindling (an animal model to induce chronic epilepsy) was induced in male Swiss albino mice by administration of pentylenetetrazole subconvulsive doses ( $35 \mathrm{mg} / \mathrm{kg}$, i.p.) at an interval of $48 \pm 2 \mathrm{~h}$ for 42 days. The epileptic animals were treated with saline; imipramine (20 mg/kg/day i.p.); fluoxetine (20 $\mathrm{mg} / \mathrm{kg} /$ day i.p.); venlafaxine (10 mg/kg/day i.p.) and mirtazapine (10 mg/kg/day i.p.) for 15 days. Except naive, animals were challenged with pentylenetetrazole subconvulsive dose $\left(35 \mathrm{mg} / \mathrm{kg}\right.$, i.p.) on every $5^{\text {th }}$ day to determine convulsion severity score, latency to first myoclonic jerk, latency to first tonic-clonic convulsions and numbers of tonic-clonic convulsions. Depression was also evaluated every $5^{\text {th }}$ day employing tail suspension test $2 \mathrm{~h}$ after pentylenetetrazole subconvulsive dose.

Results: All ADs have been reported significant antidepressant potential however regarding their safety in regard to convulsions in epileptic animals, variable results are obtained. Chronic administration of venlafaxine and mirtazapine were found to have significant anticonvulsant effect in epileptic animals. The behavioral data was further corroborated by neurochemical findings.

Received January 22, 2017 Accepted March 31, 2017

Corresponding author: Rajesh Kumar Goel Department of Pharmaceutical Sciences and Drug Research, Punjabi University, Patiala, Punjab 147002, India

Tel. + 91-9417881189

E-mail; goelrkpup@gmail.com
Conclusions: The treatment with venlafaxine and mirtazapine can be considered safe for treatment of depression in epilepsy and may enhance anticonvulsant potential of antiepileptic drugs as an adjuvant therapy. However, pharmacokinetic studies are warranted before translating these findings in PWE.

(2017;7:25-32)

Key words: Depression, Epilepsy, Fluoxetine, Imipramine, Mirtazapine, Venlafaxine

\section{Introduction}

Depression represents most debilitating psychiatric comorbidity associated with epilepsy. It increases the socioeconomic burden leading to disability and impaired quality of life in patients with epilepsy (PWE). ' Antiepileptic drugs (AEDs) such as barbiturates, benzodiazepines, tiagabine, levetiracetam ameliorate convulsions due to epilepsy but further worsens the associated depression. ${ }^{1}$ Antidepressants (ADs) administered for treatment of depression in epilepsy increases convulsion outcomes (convulsion intensity, convulsion frequency, decreases latency to convulsions) in PWE. The clinical data associated with ADs including selective serotonin reuptake inhibitors
(SSRIs) or serotonin-noradrenaline reuptake inhibitors (SNRIs), have been reported controversial for treatment of depression in epilepsy. ${ }^{2}$ The data is controversial as there are no streamline studies to evaluate the safety of different ADs in single animal model. ${ }^{3}$ Thus, definite conclusion is still lacking regarding safety of ADs in treating depression associated with epilepsy.

The main objective of our study was to be conclusive regarding the safety of four different classes of ADs. Tricyclic (TCA) class of ADs such as imipramine has been reported to block serotonin transporter (SERT) and norepinephrine transporter (NET). They also act on histamine and cholinergic receptors and have negligible dopamine transporter (DAT) affinity. The second class selected for the study was se- 
lective serotonin reuptake inhibitors (SSRIs) and this class of ADs such as fluoxetine has been reported to specifically block SERT with no additional pharmacological actions. The third class of ADs includes serotonin and noradrenaline reuptake inhibitors (SNRIs) and this class of ADs such as venlafaxine has been reported for selective SERT and NET blockade. The fourth class of ADs selected was noradrenaline and specific serotonergic antidepressant ( $\mathrm{NaSSA}$ ) and this class of ADs include mirtazapine which has been reported different mechanism of actions. NaSSA class of ADs has been reported to block a range of receptors such as $\alpha 2,5-\mathrm{HT}_{2}, 5-\mathrm{HT}_{3}$ as well as histamine 1 ( $\mathrm{H} 1)$.

The exploration of different classes of ADs in one model will minimize the experimental variables such as animal model, dose schedule, and route of drug administration etc. As the animal model employed was chronic it represents the actual clinical situation as association of depression is usually observed in patients having chronic epilepsy. ${ }^{3,4}$ Moreover, 15 days treatment of ADs will conclusively define their safety profile for the treatment of depression in epilepsy. As per literature, no study has been reported yet in which safety and efficacy has been defined after the 15 day chronic administration in chronic epileptic animals. The ADs should be safe during the whole course of therapy rather than for a short period in PWE. As per current knowledge base this will be the first study to deduce the safety of four different ADs regarding their convulsive potential in a same model of chronic epilepsy with minimum experimental variables.

ADs have been reported to inhibit the reuptake of monoamines specifically norepinephrine and serotonin as part of their complex mechanism of action. These monoamines have also been reported to possess significant anticonvulsant potential, and altered levels of these monoamines may explain either anticonvulsant or proconvulsant action for these ADs. ${ }^{3}$ Thus, we have precisely estimated serotonin and norepinephrine levels employing high performance liquid chromatography (HPLC) coupled with fluorescence detector. ${ }^{5}$

Further, in this study we employed pentylenetetrazole induced kindling model of chronic epilepsy. It is a widely explored experimental model of complex partial seizure with secondary generalization, defined by a progressive increase in electrographic and behavioral convulsions. ${ }^{6}$ Repeated administration of pentylenetetrazole subconvulsive dose results in an enhancement of convulsive responses due to plastic changes in synaptic efficacy for at least 10 months after drug discontinuation. ${ }^{7,8}$ This model is one of the few (and often the only) chronic models that are currently used by most antiepileptic discovery programs, including the National Institutes of Health/National Institute of Neurological disorders and Stroke (NIH/NINDS)-sponsored anticonvulsant screening program in the U.S. ${ }^{9}$ Depression like phenotypes are significantly associated with epileptic animals as assessed in most of our previous studies. ${ }^{10-12}$ and also reported by other researchers. ${ }^{13,14}$ Thus, the study was envisaged to report the safety of four different class of ADs i.e. imipramine, fluoxetine, venlafaxine and mirtazapine regarding their convulsive potential which will be deduced after 15 days chronic treatment at their safer antidepressant doses in pentylenetetrazole kindling induced epileptic animals associated with depression.

\section{Methods}

\section{Drugs and chemicals}

Pentylenetetrazole and serotonin was procured from Sigma-Aldrich, Co. (St. Louis, MO, USA). HPLC-grade methanol and heptane sulfonic acid was procured from Merck Specialties (Mumbai, India). Norepinephrine was obtained as a gift sample from Troikaa Pharmaceuticals Ltd. (Dehradun, India). Perchloric acid was purchased from Spectrochem (Mumbai, India). Imipramine hydrochloride, fluoxetine hydrochloride, venlafaxine hydrochloride and mirtazapine were obtained as gift samples from Parasol Laboratories, Baddi, Himachal Pradesh (India).

\section{Animals}

The study was carried out in two months old Swiss albino male mice weighing 22-28 g, obtained from Lala Lajpat Rai University of Veterinary and Animal Sciences, Hisar, Haryana. The animals were housed in groups of 6 mice/cage at an ambient room temperature of $22 \pm 2^{\circ} \mathrm{C}$ and relative humidity of $50 \pm 5 \%$ and maintained under a 12:12 h dark light cycle (lights on at 07:00 h). Food and water were available ad libitum except during specific experimental protocols. The experimental protocol was duly approved by the Institutional Animal Ethics Committee (IAEC) and the care of the animals was carried out as per the guidelines of the Committee for the Purpose of Control and Supervision of Experiments on Animals (CPCSEA), Ministry of Environment and Forest, Government of India vide protocol approval no. 107/99/CPCSEA-2010-18.

\section{Epilepsy/kindling induction}

Kindling was carried out by the method previously validated in our laboratory. ${ }^{10-12}$ The pentylenetetrazole (dissolved in saline) was injected intraperitoneally in a volume of $10 \mathrm{~mL} / \mathrm{kg}$ mice at a sub-con- 
vulsive dose of $35 \mathrm{mg} / \mathrm{kg}$ at $48 \pm 2 \mathrm{~h}$ intervals for 42 days. After each injection, the mice were placed individually into plexiglass cages (20 $\times 20 \times 30 \mathrm{~cm}$ ) and observed for $30 \mathrm{~min}$. The intensity of the convulsions was registered according to modified racine's scale; stage 0 : no response; stage 1: hyperactivity, restlessness and vibrissae twitching; stage 2: head nodding, head clonus and myoclonic jerks; stage 3: continuous myoclonic jerk with tail rigidity; stage 4: generalized limbic seizures with kangaroo posture or violent convulsions; stage 5: generalized tonic-clonic seizures with falling and stage 6: hind limb extensor. The transition of the convulsion intensity from the $4^{\text {th }}$ to $5^{\text {th }}$ stage reflected the generalization of the convulsive activity, manifested by the tonic-clonic convulsions. The animals were considered kindled after five times appearance of stage 5 (tonic-clonic convulsions) seizures on consecutive subconvulsive pentylenetetrazole administrations during kindling.

\section{Experimental protocol}

Total 46 animals were employed in this study. The Group I: NAIVE, consisted of naive animals (non-kindled, $n=6$ ) and rest 40 animals were subjected to pentylenetetrazole kindling. The animals developing epilepsy ( $n=30)$, were divided into five groups (Group II to VI), namely Group II: SAL, saline treated kindled animals ( $\mathrm{n}=$ 6), Group III: IMI, imipramine (20 mg/kg/day i.p.) treated kindled animals $(n=6)$, Group IV: fluoxetine (20 mg/kg/day i.p.) treated kindled animals $(n=6)$, Group V: venlafaxine (10 mg/kg/day i.p.) treated kindled animals $(n=6)$ and Group Vl: mirtazapine (10 $\mathrm{mg} / \mathrm{kg} /$ day i.p.) treated kindled animals $(n=6)$. From the very next day after the last pentylenetetrazole injection, the treatment was initiated and it was considered as day 1. All the groups except naive were given treatment for 15 days. On every $5^{\text {th }}$ day during treatment, Groups II-VI were challenged with a subconvulsive dose of pentylenetetrazole $(35 \mathrm{mg} / \mathrm{kg}$; i.p.) $30 \mathrm{~min}$ after their respective treatments, and the convulsion severity score, latency to first myoclonic jerk, latency to first tonic-clonic convulsions and number of tonic-clonic convulsions were observed for $30 \mathrm{~min}$. Two hours after pentylenetetrazole injection, animals were subjected to tail suspension test (after confirming the normalization of locomotor activity). On the $15^{\text {th }}$ day of treatment, $4 \mathrm{~h}$ after behavioral assessment (for excluding any potential effect of behavioral estimations on neurochemical changes), all animals were immediately euthanized and the brain was separated in cold room (maintained at $4^{\circ} \mathrm{C}$ ) for neurochemical analysis. All these experiments were carried out in the same time of the day to exclude any significant effect of circadian

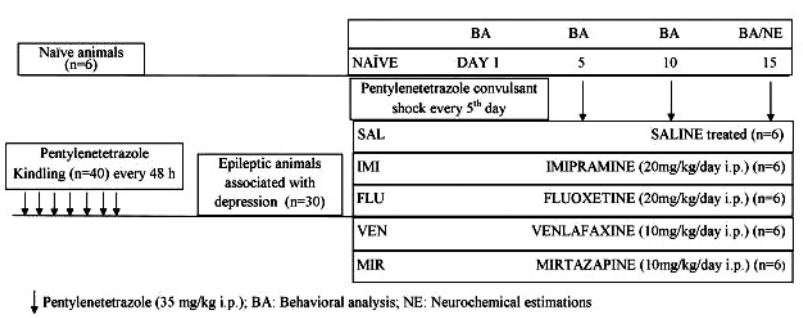

Figure 1. Schematic presentation of experimental protocol.

rhythm on altered neurochemical milieu (Fig. 1).

\section{Behavioral analysis}

\section{Tail suspension test}

The tail suspension test was employed to estimate immobility as an index of depression associated with animals. The method is based on the observations that a mouse suspended by the tail shows alternating periods of mobility and immobility (mobility reflects efforts to correct the posture, and immobility reflects despair). The whole session was recorded for 6 min and immobility time was calculated. An animal was considered to be immobile when it did not show any body movements and hung passively. ${ }^{15}$

\section{Neurochemical analysis}

Neurochemical estimations were carried out to assess if there is any correlation between decreased convulsion outcomes with total nitrite levels, serotonin, and norepinephrine levels in the brain. Isolated brain was weighed and subdivided into two equal portions. One portion was homogenized in ice cold $10 \% \mathrm{w} / \mathrm{v}(0.1 \mathrm{M})$ perchloric acid and centrifuged at $14,000 \mathrm{~g}$ for $30 \mathrm{~min}$ at $4^{\circ} \mathrm{C}$ (REMI C-24BL, Cooling Centrifuge, REMI, India) and clear supernatant separated was used for the estimation of serotonin and norepinephrine. While second portion was homogenized in ice cold $10 \% \mathrm{w} / \mathrm{v}(0.05 \mathrm{M}, \mathrm{pH}$ 7.4) phosphate buffer and centrifuged at $6,000 \mathrm{~g}$ for $20 \mathrm{~min}$ at $4^{\circ} \mathrm{C}$ and clear supernatant was utilized for estimation of total nitrite level estimations using microplate reader method. ${ }^{5}$

\section{Estimation of norepinephrine and serotonin}

The estimation of norepinephrine and serotonin was carried out employing HPLC fluorometric method, previously standardized in our laboratory. ${ }^{5}$ The mobile phase consisted of sodium acetate $(0.02 \mathrm{M})$, methanol (16\%), heptane sulfonic acid $(0.137 \%)$, EDTA $(0.2 \mathrm{mM})$, and dibutylamine $(0.1 \% \mathrm{v} / \mathrm{v})$. The solution was adjusted to $\mathrm{pH} 3.92 \pm$ 0.01 with o-phosphoric acid and filtered through a $0.45 \mu \mathrm{m}$ mem- 
brane (Millipore, USA) and degassed (Transonic T 570/H, Elma, Germany). The flow rate was set to $1 \mathrm{~mL} / \mathrm{min}$. Norepinephrine and serotonin was detected at the excitation wavelength $280 \mathrm{~nm}$ and emission wavelength of $315 \mathrm{~nm}$. The straight line equation was derived (norepinephrine: $y=124373 x+255920, R^{2}=0.9981$; serotonin: $y=151725 x-446,462 ; R^{2}=0.997$; where $x$ : concentration in $\mathrm{ng} / \mathrm{ml}$ and $\mathrm{y}$ : area under peak). The results were expressed as percentage in comparison to naive animals (Norepinephrine and serotonin levels in naïve is considered as 100\%).

\section{Estimation of total nitrite levels}

The estimation of nitrosative stress in the form of total nitrite levels was carried out as per method previously standardized. ${ }^{16}$ The method involves the use of the Griess diazotization reaction to spectrophotometrically detect total nitrite levels. In this method, $50 \mu \mathrm{L}$ of brain homogenate (filtered) and the standard $\left(\mathrm{NaNO}_{2} / \mathrm{NaNO}_{3}\right)$ was mixed with $50 \mu \mathrm{L}$ of the Griess reagent in triplicate using a 96-well plate. In the supernatant well, $\mathrm{Cu} / \mathrm{Cd}$ alloy was added to ensure the conversion of nitrates to nitrites. The 96-well plate was shaken at 150 RPM for 1 min to ensure proper mixing of the samples/standard with the Griess reagent. The plate was incubated for $30 \mathrm{~min}$ at room temperature. The absorbance of nitrite-containing samples was measured at $540 \mathrm{~nm}$ using a microplate reader (APR-4 Microplate Reader, Logotech, ISE Group, Germany) against a photometric reference (blank: $50 \mu \mathrm{L} \mathrm{HPLC}$ grade water and $50 \mu \mathrm{L}$ Griess Reagent). The total nitrite levels were estimated using the straight line equation for nitrite $(y=0.0008 x+0.0046, r 2=0.9954)$. The results were expressed as $n g / g$ of wet tissue.

\section{Statistical analysis}

The statistical analysis was performed using the Graph Pad Prism version 3.5. For behavioral estimations, comprising of two variables (different treatments and different days), the intergroup variation was measured by two-way analysis of variance (ANOVA) followed by Bonferroni's test (for multivariate analysis), while for the neurochemical estimations (comprising of one variable i.e. different treatment), a one-way ANOVA followed by Tukey's test was applied. The results were expressed as mean \pm standard error mean and statistical significance was considered at $p<0.05$.

\section{Results}

\section{Epilepsy/kindling induction}

The average number of pentylenetetrazole injections required to induce a successful epileptic condition in mice were found to be $16 \pm$ 4. Ten animals were found resistant to develop epilepsy and were not employed in the study.

\section{Behavioral results}

Effect of different pharmacological interventions on immobility time

A significant increase $(p<0.05)$ in immobility time was observed in saline treated kindled animals on days 1, 5, 10, and 15 as compared to naive. Treatment with imipramine, fluoxetine, venlafaxine and mirtazapine significantly $(p<0.05)$ reduced the immobility time on days 5, 10, and 15 as compared to saline treated kindled animals (Fig. 2).

Effect of different pharmacological interventions on convulsion severity score

Treatment with imipramine, fluoxetine, venlafaxine and mirtazapine did not have significant $(p<0.05)$ effect on convulsion severity score on day 5 as compared to saline treated kindled animals.

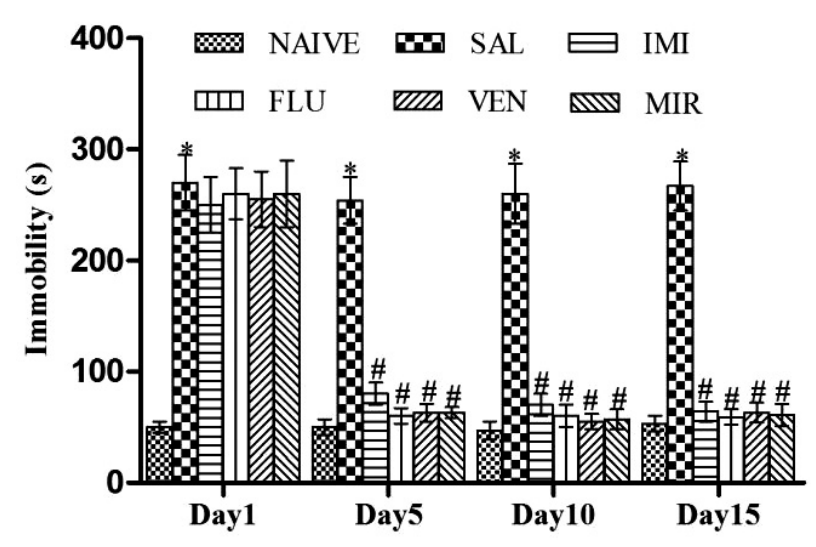

Figure 2. Effect of different pharmacological interventions on the immobility period in the tail suspension test. Each value is expressed as mean \pm standard error mean. *As compared to naive; ${ }^{*}$ as compared to saline treated kindled animals. The significance level was considered at $p<$ 0.05 (Bonferroni's Test). SAL, saline treated kindled animals; IMI, imipramine (20 mg/kg i.p./day) treated kindled animals; FLU, fluoxetine (20 $\mathrm{mg} / \mathrm{kg}$ i.p./day) treated kindled animals; VEN, venlafaxine $(10 \mathrm{mg} / \mathrm{kg}$ i.p./day) treated kindled animals; MIR, mirtazapine (10 mg/kg i.p./day) treated kindled animals. 


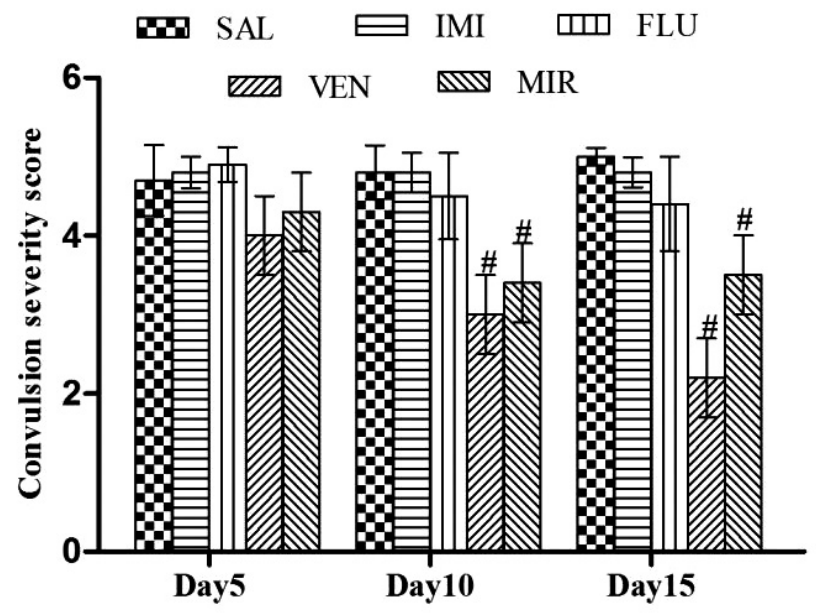

Figure 3. Effect of different pharmacological interventions on convulsion severity score. Each value is expressed as mean \pm standard error mean. \#As compared to saline treated kindled animals. The significance level was considered at $p<0.05$ (Bonferroni's Test). SAL, saline treated kindled animals; IMl, imipramine (20 mg/kg i.p./day) treated kindled animals; FLU, fluoxetine (20 mg/kg i.p./day) treated kindled animals; VEN, venlafaxine (10 $\mathrm{mg} / \mathrm{kg}$ i.p./day) treated kindled animals; MIR, mirtazapine $(10 \mathrm{mg} / \mathrm{kg}$ i.p./day) treated kindled animals.

Treatment with imipramine and fluoxetine did not have significant effect on convulsion severity score on days 10 and 15 as compared to saline treated kindled animals. However, treatment with venlafaxine and mirtazapine significantly $(p<0.05)$ reduced the convulsion severity score on days 10 and 15 as compared to saline treated kindled animals (Fig. 3).

Effect of different pharmacological interventions on latency to first myoclonic jerks

No significant $(p<0.05)$ decrease in latency to first myoclonic jerk was observed in any of the antidepressant treated group in comparison to saline treated kindled animals on day 5 . On days 10 and 15 , imipramine significantly $(p<0.05)$ decreased latency to first myoclonic jerk whereas in venlafaxine and mirtazapine treated kindled animals significantly $(p<0.05)$ increased latency to first myoclonic jerk was observed as compared to saline treated kindled animals. However, fluoxetine was not reported any significant $(p<0.05)$ effect on myoclonic jerk as compared to saline treated kindled animals (Fig. 4).

Effect of different pharmacological interventions on latency to first tonic-clonic convulsions

There was no significant difference observed in latency to first tonic-clonic convulsions in different antidepressant treatment groups as

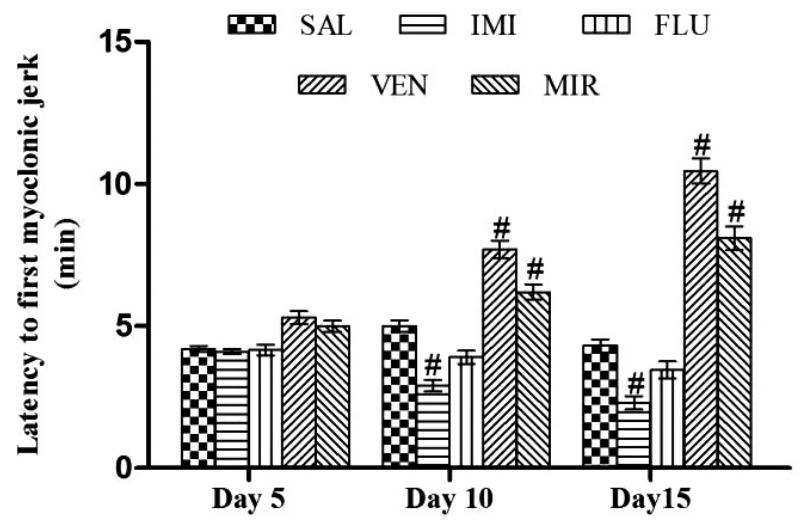

Figure 4. Effect of different pharmacological interventions on latency to first myoclonic jerk. Each value is expressed as mean \pm standard error mean. \#As compared to saline treated kindled animals. The significance level was considered at $p<0.05$ (Bonferroni's Test). SAL, saline treated kindled animals; IMl, imipramine (20 mg/kg i.p./day) treated kindled animals; FLU, fluoxetine (20 mg/kg i.p./day) treated kindled animals; VEN, venlafaxine (10 mg/kg i.p./day) treated kindled animals; MIR, mirtazapine (10 mg/kg i.p./day) treated kindled animals.

compared to saline treated kindled animals on day 5. On day 10, imipramine significantly $(p<0.05)$ decreased latency to first tonic-clonic convulsions whereas venlafaxine significantly $(p<0.05)$ increased the latency to first tonic-clonic convulsions as compared to saline treated kindled animals. On day 15, imipramine significantly ( $p$ $<0.05)$ decreased latency to first tonic-clonic convulsions whereas venlafaxine and mirtazapine significantly $(p<0.05)$ increased latency as compared to saline treated kindled animals. However, no significant $(p<0.05)$ effect of fluoxetine on latency to first tonic-clonic convulsions was observed as compared to saline treated kindled animals (Fig. 5).

Effect of different pharmacological interventions on number of tonic-clonic convulsions

The treatment with imipramine and fluoxetine treatment significantly $(p<0.05)$ increased number of tonic-clonic convulsions on days 5,10 , and 15 as compared to saline treated kindled animals, but no significant increase was observed with mirtazapine and venlafaxine treatment on days 5, 10, and 15 (Fig. 6).

\section{Neurochemical results}

Effect of different pharmacological interventions on norepinephrine levels

The significant $(p<0.05)$ depletion in norepinephrine levels was observed in saline treated kindled animals as compared to naive 


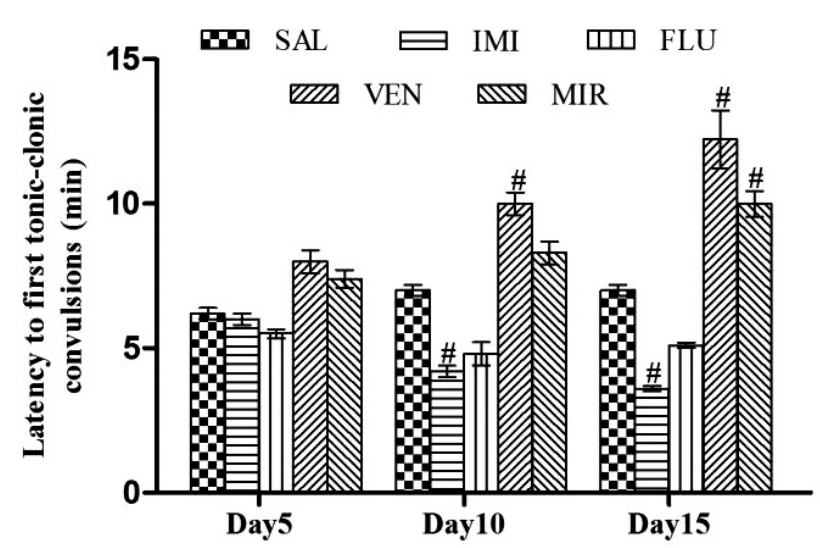

Figure 5. Effect of different pharmacological interventions on latency to first tonic-clonic convulsions. Each value is expressed as mean \pm standard error mean. \#As compared to saline control kindled animals. The significance level was considered at $p<0.05$ (Bonferroni's Test). SAL, saline treated kindled animals; IMl, imipramine (20 mg/kg i.p./day) treated kindled animals; FLU, fluoxetine ( $20 \mathrm{mg} / \mathrm{kg}$ i.p./day) treated kindled animals; VEN, venlafaxine (10 $\mathrm{mg} / \mathrm{kg}$ i.p./day) treated kindled animals; MIR, mirtazapine $(10 \mathrm{mg} / \mathrm{kg}$ i.p./day) treated kindled animals.

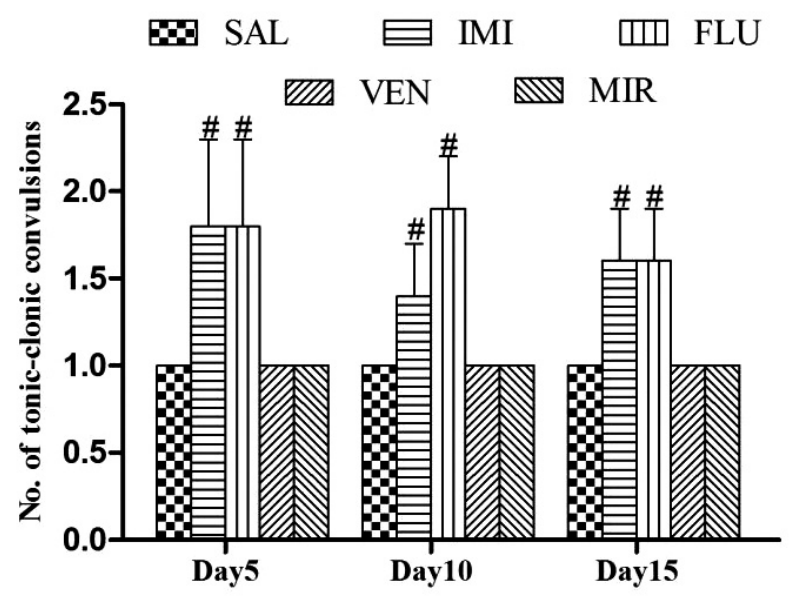

Figure 6. Effect of different pharmacological interventions on number of tonic-clonic convulsions. Each value is expressed as mean \pm standard error mean. \#As compared to saline treated kindled animals. The significance level was considered at $p<0.05$ (Bonferroni's Test). SAL, saline treated kindled animals; IMI, imipramine (20 mg/kg i.p./day) treated kindled animals; FLU, fluoxetine (20 mg/kg i.p. /day) treated kindled animals; VEN, venlafaxine (10 mg/kg i.p./day) treated kindled animals; MIR, mirtazapine (10 mg/kg i.p./day) treated kindled animals.

Table 1. Effect of different pharmacological interventions on norepinephrine, serotonin and total nitrite levels

\begin{tabular}{lccc}
\hline Analytes & Norepinephrine & Serotonin & Total nitrite levels \\
\hline Group & $100 \pm 6.63$ & $100 \pm 14.17$ & $100 \pm 0.35$ \\
NAIVE & $29.4 \pm 5.61^{*}$ & $27.2 \pm 2.48^{\star}$ & $475.38 \pm 0.34^{\star}$ \\
SAL & $106.48 \pm 4.56^{\#}$ & $119.54 \pm 12.34^{\#}$ & $301.54 \pm 0.38^{* \#}$ \\
IMI & $94.56 \pm 6.49^{\#}$ & $140.17 \pm 17.01^{\#}$ & $290.77 \pm 0.25^{\star \#}$ \\
FLU & $159.03 \pm 7.88^{* \#}$ & $203.65 \pm 19.56^{* \#}$ & $25.38 \pm 0.10^{* \#}$ \\
VEN & $179.16 \pm 8.54^{* \#}$ & $196.90 \pm 22.45^{* \#}$ & $90.77 \pm 0.25^{\#}$ \\
\hline MIR
\end{tabular}

Each value is expressed as mean \pm standard error mean. Naive group was considered as $100 \%$.

*As compared to naive; "as compared to saline treated kindled animals. The significance level was considered at $p<0.05$ (Tukey's Test). SAL, saline treated kindled control animals; IMl, imipramine (20 mg/kg i.p./day) treated kindled animals; FLU, fluoxetine (20 mg/kg i.p./day) treated kindled animals; VEN, venlafaxine (10 mg/kg i.p./day) treated animals; MIR, mirtazapine (10 mg/kg i.p./day) treated animals.

(Naive norepinephrine Level is considered as 100\%). The treatment with imipramine, fluoxetine, venlafaxine and mirtazapine significantly $(p<0.05)$ increased percentage brain norepinephrine level in comparison to saline treated kindled animals as shown in Table 1.

Effect of different pharmacological interventions on serotonin levels

The significant $(p<0.05)$ reduction in serotonin levels was observed in saline treated kindled animals as compared to naive (Naive serotonin Level is considered as $100 \%)$. The treatment with imipramine, fluoxetine, venlafaxine and mirtazapine significantly $(p<$
0.05) increased percentage brain serotonin level in comparison to saline treated kindled animals as shown in Table 1.

\section{Effect of different pharmacological interventions on total} nitrite levels

The significant $(p<0.05)$ increase in percentage total nitrite levels was observed in saline treated kindled animals as compared to naive (Naive brain total nitrite level is considered as 100\%). The treatment with imipramine, fluoxetine, venlafaxine and mirtazapine significantly $(p<0.05)$ reduced percentage total nitrite levels as compared to saline treated kindled animals as shown in Table 1. 


\section{Discussion}

This study was envisaged to investigate the relative safety of four different classes of ADs regarding their convulsive potential at their safer antidepressant dose in chronic epileptic animals associated with depression. All four ADs have been reported significant antidepressant effect demonstrating their efficacy in treating the depression associated with epilepsy. Elevated norepinephrine and serotonin levels may explain potent antidepressant potential of these ADs. However, among four different classes of antidepressants, venlafaxine and mirtazapine were found to decrease convulsion outcomes (convulsion severity score, latency to first myoclonic jerk, latency to first tonic-clonic convulsion and number of tonic-clonic convulsions) in chronic epileptic animals.

The chronic treatment with imipramine lowered the convulsion threshold as evident by its propensity to decrease latency to first myoclonic jerk and first tonic-clonic convulsions with elevated frequency of tonic-clonic convulsions in 30 min duration after convulsive shock. The convulsive potential of imipramine had also been reported earlier. ${ }^{17-19}$ Although elevated norepinephrine and serotonin levels may constitute towards anticonvulsant effect of imipramine, ${ }^{3}$ but overall we observed proconvulsant effect. Increased nitrosative stress in imipramine treated epileptic animal, observed in the study with reported inhibitory effect on GABA receptors and histamine $\mathrm{H} 1$ receptors may explain proconvulsant effect observed for imipramine. $^{18-20}$

Chronic treatment with fluoxetine did not have significant effect on convulsion outcomes except tonic-clonic convulsion frequency which was observed to be increased. These findings were further corroborated by clinical findings. ${ }^{20-21}$ Elevated nitrosative stress may explain the elevated convulsion frequency observed with fluoxetine treatment in epileptic animals. ${ }^{20}$ The proconvulsant effect for chronic fluoxetine could be due to either increased firing of serotonergic neurons due to down regulation of presynaptic $5-\mathrm{HT}_{1 \mathrm{~A}}$ receptors or due to down regulation of post-synaptic $5-\mathrm{HT}_{2}$ receptors. ${ }^{22}$

Chronic treatment with venlafaxine and mirtazapine in epileptic animals was found significant anticonvulsant effect. These findings were corroborated by previous findings. ${ }^{23,24}$ The anticonvulsant potential of venlafaxine and mirtazapine may be explained by elevated serotonin and norepinephrine levels (nearly 100\% elevation was observed) in comparison to naive animals. The nitrosative stress was also found to be restored as observed in naive animals, pertaining to potent anti-inflammatory potential of both mirtazapine and venlafaxine. ${ }^{25,26}$ Elevated serotonin and norepinephrine levels as well as decreased nitrosative stress may justify the anticonvulsant effects of venlafaxine and mirtazapine. Further, venlafaxine have been reported to activate $5-\mathrm{HT}_{1 \mathrm{~A}}, 5-\mathrm{HT}_{1 \mathrm{~B}}$ and $\beta 2$ receptors and elevate the allopregnanolone levels which may complement its anticonvulsant potential. ${ }^{27,28}$ Similarly, mirtazapine in addition to block monoamine reuptake, mediates anticonvulsant action through antagonism of $5-\mathrm{HT}_{2 \mathrm{~A} 2 \mathrm{C}}$ receptors. ${ }^{29}$

In conclusion, the behavioral analysis confirmed significant antidepressant potential for all classes of ADs in chronic epileptic animals. Regarding their convulsive potential, venlafaxine and mirtazapine have better safety. However, pharmacokinetic interaction studies with confirmation in another model of epilepsy are necessary before translating these findings for treatment of depression in PWE.

\section{Acknowledgements}

The authors are grateful to the Department of Pharmaceutical Sciences and Drug Research, Punjabi University, Patiala for providing funding and necessary technical facilities for present research work.

\section{Conflict of Interest}

The authors report no conflicts of interest.

\section{References}

1. Kanner AM. Management of psychiatric and neurological comorbidities in epilepsy. Nat Rev Neurol 2016;12:106-16.

2. Maguire MJ, Weston J, Singh J, Marson AG. Antidepressants for people with epilepsy and depression. Cochrane Database Syst Rev 2014;12:CD010682.

3. Cardamone L, Salzberg MR, O'Brien TJ, Jones NC. Antidepressant therapy in epilepsy: can treating the comorbidities affect the underlying disorder? Br J Pharmacol 2013;168:1531-54.

4. Igelstrom KM. Preclinical antiepileptic actions of selective serotonin reuptake inhibitors - implications for clinical trial design. Epilepsia 2012:53:596-605.

5. Singh D, Mishra A, Goel RK. Effect of saponin fraction from Ficus religiosa on memory deficit, and behavioral and biochemical impairments in pentylenetetrazol kindled mice. Epilepsy Behav 2013;27:206-11.

6. Morimoto K, Fahnestock M, Racine RJ. Kindling and status epilepticus models of epilepsy: rewiring the brain. Prog Neurobio/ 2004;73:1-60.

7. Diehl RG, Smialowski A, Gotwo T. Development and persistence of kindled seizures after repeated injections of pentylenetetrazol in rats 
and guinea pigs. Epilepsia 1984;25:506-10.

8. Corda MG, Orlandi $M$, Lecca $D$, et al. Pentylenetetrazol induced kindling in rats: effect of GABA function inhibitors. Pharmacol Biochem Behav 1991;40:329-33.

9. Löscher W. Fit for purpose application of currently existing animal models in the discovery of novel epilepsy therapies. Epilepsy Res 2016;126:157-84.

10. Singh T, Goel R.K. Evidence in support of using a neurochemistry approach to identify therapy for both epilepsy and associated depression. Epilepsy Behav 2016;61:248-57.

11. Singh T, Goel RK. Adjuvant indoleamine 2,3-dioxygenase enzyme inhibition for comprehensive management of epilepsy and comorbid depression. Eur J Pharmacol 2016b;784:111-20.

12. Singh T, Goel RK. Adjuvant neuronal nitric oxide synthase inhibition for combined treatment of epilepsy and comorbid depression. Pharmacol Rep 2017;69:143-9.

13. Mortazavi F, Ericson M, Story D, Hulce VD, Dunbar GL. Spatial learning deficits and emotional impairments in pentylenetetrazole-kindled rats. Epilepsy Behav 2005;7:629-38.

14. Russo E, Chimirri $S$, Aiello $R$, et al. Lamotrigine positively affects the development of psychiatric comorbidity in epileptic animals, while psychiatric comorbidity aggravates seizures. Epilepsy Behav 2013;28:232-40.

15. Steru $L$, Chermat R, Thierry B, Simon P. The tail suspension test: a new method for screening antidepressants in mice. Psychopharmacology (Berl) 1985;85:367-70.

16. Singh T, Goel RK. Neuroprotective effect of Allium cepa L. in aluminium chloride induced neurotoxicity. Neurotoxicology 2015;49:1-7.

17. Koella WP, Glatt A, Klebs K, Dürst T. Epileptic phenomena induced in the cat by the antidepressants maprotiline imipramine clomipramine and amitriptyline. Biol Psychiatry 1979;14:485-97.

18. Ago J, Ishikawa T, Matsumoto $N$, Ashequr Rahman M, Kamei C.
Mechanism of imipramine induced seizures in amygdala-kindled rats. Epilepsy Res 2006;72:1-9.

19. Ago J, Ishikawa T, Matsumoto N, Rahman MA, Kamei C. Epileptiformic activity induced by antidepressants in amygdala-kindled rats. Eur J Pharmacol 2007;560:23-8.

20. Shin EJ, Jeong JH, Chung $\mathrm{YH}$, et al. Role of oxidative stress in epileptic seizures. Neurochem Int 2011;59:122-37.

21. Gigli GL, Diomedi M, Troisi A, et al. Lack of potentiation of anticonvulsant effect by fluoxetine in drug-resistant epilepsy. Seizure 1994;3:221-4.

22. Raju SS, Noor AR, Gurthu $S$, et al. Effect of fluoxetine on maximal electroshock seizures in mice: acute vs chronic administration. Pharmacol Res 1999;39:451-4.

23. Fawcett J, Barkin RL. Review of the results from clinical studies on the efficacy, safety and tolerability of mirtazapine for the treatment of patients with major depression. J Affect Disord 1998;51:267-85.

24. Montgomery SA. Antidepressants and seizures: emphasis on newer agents and clinical implications. Int J Clin Pract 2005;59:1435-40.

25. Altuner D, Gulaboglu M, Yapca OE, Cetin N. The effect of mirtazapine on cisplatin-induced oxidative damage and infertility in rat ovaries. ScientificWorldJournal 2013;2013:327240.

26. Hajhashemi V, Minaiyan M, Banafshe HR, Mesdaghiia A, Abed A. The anti-inflammatory effects of venlafaxine in the rat model of carrageenan-induced paw edema. Iran J Basic Med Sci 2015;18:654-8.

27. Bourin M. Psychopharmacological profile of venlafaxine. Encephale 1999;25:21-2; discussion 23-5.

28. Uzunova V, Wrynn AS, Kinnunen A, et al. Chronic antidepressants reverse cerebrocortical allopregnanolone decline in the olfactory bulbectomized rat. Eur J Pharmacol 2004;486:31-4.

29. Wada Y, Nakamura M, Hasegawa H, Yamaguchi N. Role of serotonin receptor subtype in seizures kindled from the feline hippocampus. Neurosci Lett 1992;141:21-4. 